\title{
SUBADDITIVITY OF HOMOGENEOUS NORMS ON CERTAIN NILPOTENT LIE GROUPS
}

\author{
JACEK CYGAN
}

\begin{abstract}
ABSTRACr. Let $N$ be a Lie group with its Lie algebra generated by the leftinvariant vector fields $X_{1}, \ldots, X_{k}$ on $N$. An explicit fundamental solution for the (hypoelliptic) operator $L=X_{1}^{2}+\cdots+X_{k}^{2}$ on $N$ has been obtained for the Heisenberg group by Folland [1] and for the nilpotent (Iwasawa) groups of isometries of rank-one symmetric spaces by Kaplan and Putz [2]. Recently Kaplan [3] introduced a (still larger) class of step-2 nilpotent groups $N$ arising from Clifford modules for which similar explicit solutions exist. As in the case of $L$ being the ordinary Laplacian on $N=\mathbf{R}^{k}$, these solutions are of the form $g \mapsto$ const $\|g\|^{2-m}$, $g \in N$, where the "norm" function \| $\|$ satisfies a certain homogeneity condition. We prove that the above norm is also subadditive.
\end{abstract}

Let $\mathfrak{u}, \mathfrak{v}$ be real finite-dimensional vector spaces each equipped with a positive definite quadratic form ||$^{2}$. Let $\mu: \mathfrak{u} \times \mathfrak{v} \rightarrow \mathfrak{b}$ be a composition of these quadratic forms [3, p. 148] normalized in the sense that $\mu\left(u_{0}, v\right)=v$ for some $u_{0} \in \mathfrak{u}$. Define $\phi: \mathfrak{b} \times \mathfrak{b} \rightarrow \mathfrak{u}$ by demanding $\left\langle u, \phi\left(v, v^{\prime}\right)\right\rangle=\left\langle\mu(u, v), v^{\prime}\right\rangle, u \in \mathfrak{u} ; v, v^{\prime} \in \mathfrak{b}$, relative to the inner products $\langle$,$\rangle induced by the given quadratic forms. Let z$ denote the orthogonal complement to $\mathbf{R} u_{0}$ in $\mathfrak{u}$ and $\pi: \mathfrak{u} \rightarrow \mathfrak{z}$ the orthogonal projection. Now set $\mathfrak{n}=\mathfrak{b} \times \mathfrak{z}$ and define a bracket on $\mathfrak{n}$ by $\left[(v, z),\left(v^{\prime}, z^{\prime}\right)\right]=\left(0, \pi \circ \phi\left(v, v^{\prime}\right)\right)$. On the simply connected analytic group $N$, corresponding to the Lie algebra $\mathfrak{n}$ (i.e. on Kaplan's type $\mathbf{H}$ group) we define a norm function by $\|n\|=\left(|v|^{4}+16|z|^{2}\right)^{1 / 4}$, where $n=\exp (v+z), v \in \mathfrak{b}, z \in \mathfrak{z} ; \mathfrak{n} \cong \mathfrak{v} \oplus \mathfrak{z}$. We now prove

THEOREM. The norm function \|| $\|$ is subadditive, i.e.

$$
\left\|n n^{\prime}\right\| \leqslant\|n\|+\left\|n^{\prime}\right\|, \quad n, n^{\prime} \in N .
$$

Proof. We have

$$
\begin{aligned}
\left\|n n^{\prime}\right\|^{4} & =\left\|\exp \left(v+v^{\prime}+z+z^{\prime}+\frac{1}{2}\left[v, v^{\prime}\right]\right)\right\|^{4} \\
& =\left|v+v^{\prime}\right|^{4}+16\left|z+z^{\prime}+\frac{1}{2}\left[v, v^{\prime}\right]\right|^{2}
\end{aligned}
$$

Now

$$
\begin{aligned}
\left|v+v^{\prime}\right|^{4}= & |v|^{4}+\left|v^{\prime}\right|^{4}+4\left\langle v, v^{\prime}\right\rangle^{2}+4|v|^{2}\left\langle v, v^{\prime}\right\rangle \\
& +4\left|v^{\prime}\right|^{2}\left\langle v, v^{\prime}\right\rangle+2|v|^{2}\left|v^{\prime}\right|^{2},
\end{aligned}
$$

Received by the editors March 24, 1980 and, in revised form, September 15, 1980 and November 5, 1980.

AMS (MOS) subject classifications (1970). Primary 43A80, 22E15; Secondary 35C05, 35 H05.

Key words and phrases. Analysis on nilpotent groups, gauges and homogeneous norms, (analytic-) hypoelliptic operators. 


$$
\begin{aligned}
16\left|z+z^{\prime}+\frac{1}{2}\left[v, v^{\prime}\right]\right|^{2}= & 16|z|^{2}+16\left|z^{\prime}\right|^{2}+4\left|\left[v, v^{\prime}\right]\right|^{2} \\
& +16\left\langle z,\left[v, v^{\prime}\right]\right\rangle+16\left\langle z^{\prime},\left[v, v^{\prime}\right]\right\rangle+32\left\langle z, z^{\prime}\right\rangle .
\end{aligned}
$$

Since $2|v|^{2}\left|v^{\prime}\right|^{2}+32\left\langle z, z^{\prime}\right\rangle\left\langle 2\|n\|^{2}\left\|n^{\prime}\right\|^{2}\right.$ and

$$
4|v|^{2}\left\langle v, v^{\prime}\right\rangle+16\left\langle z,\left[v, v^{\prime}\right]\right\rangle\left\langle 4\|n\|^{2}\left(\left\langle v, v^{\prime}\right\rangle^{2}+\left|\left[v, v^{\prime}\right]\right|^{2}\right)^{1 / 2},\right.
$$

we need

LEMMA. In the notation above

$$
\left\langle v, v^{\prime}\right\rangle^{2}+\left|\left[v, v^{\prime}\right]\right|^{2}\left\langle|v|^{2}\left|v^{\prime}\right|^{2}, \quad v, v^{\prime} \in \mathfrak{v} .\right.
$$

For we have $|v|\left|v^{\prime}\right| \leqslant\|n\|\left\|n^{\prime}\right\|$, and collecting the above inequalities we obtain (1) $+(2)<\left(\|n\|+\left\|n^{\prime}\right\|\right)^{4}$.

Proof of the Lemma follows from Schwarz's inequality on the hermitian form $h_{z}$ on $\mathfrak{b}$ defined by

$$
h_{z}\left(v, v^{\prime}\right)=\left\langle v, v^{\prime}\right\rangle-\sqrt{-1}\left\langle z, \pi \circ \phi\left(v, v^{\prime}\right)\right\rangle,
$$

if one regards $\mathfrak{v}$ as a complex vector space under the complex structure $J_{z}: \mathfrak{v} \rightarrow \mathfrak{v}$ given by $\left\langle J_{z}(v), v^{\prime}\right\rangle=\left\langle z, \phi\left(v, v^{\prime}\right)\right\rangle$ with fixed $z \in z,|z|=1$ (see [3, pp. 149, 150]), simply by putting $z=\left[v, v^{\prime}\right] /\left[\left[v, v^{\prime}\right] \mid\right.$.

The initial proof of the Lemma was modernized by the referee to whom I am very grateful.

\section{REFERENCES}

1. G. B. Folland, A fundamental solution for a subelliptic operator, Bull. Amer. Math. Soc. 79 (1973), 373-376.

2. A. Kaplan and R. Putz, Boundary behavior of harmonic forms on a rank one symmetric space, Trans. Amer. Math. Soc. 231 (1977), 369-384.

3. A. Kaplan, Fundamental solutions for a class of hypoelliptic PDE generated by compasition of quadratic forms, Trans. Amer. Math. Soc. 258 (1980), 147-153.

InstTtute of Mathematics, University of Wrockaw, Wrockaw, Poland 